\title{
PAPER
}

\section{FMRI studies of associative encoding in young and elderly controls and mild Alzheimer's disease}

\author{
R A Sperling, J F Bates, E F Chua, A J Cocchiarella, D M Rentz, B R Rosen, D L Schacter, \\ M S Albert
}

J Neurol Neurosurg Psychiatry 2003;74:44-50

See end of article for authors' affiliations

......................

Correspondence to: Dr Reisa Sperling, 221 Longwood Avenue, Boston, MA 02115, USA; reisa@rics.bwh.harvard.ed

Received 4 March 2002 In revised form 22 August 2002

Accepted 12 September 2002

\begin{abstract}
Objective: To examine alterations in patterns of brain activation seen in normal aging and in mild Alzheimer's disease by functional magnetic resonance imaging (fMRI) during an associative encoding task.

Methods: 10 young controls, 10 elderly controls, and seven patients with mild Alzheimer's disease were studied using fMRI during a face-name association encoding task. The fMRI paradigm used a block design with three conditions: novel face-name pairs, repeated face-name pairs, and visual fixation.

Results: The young and elderly controls differed primarily in the pattern of activation seen in prefrontal and parietal cortices: elderly controls showed significantly less activation in both superior and inferior prefrontal cortices but greater activation in parietal regions than younger controls during the encoding of novel face-name pairs. Compared with elderly controls, the Alzheimer patients showed significantly less activation in the hippocampal formation but greater activation in the medial parietal and posterior cingulate regions.
\end{abstract}

Conclusions: The pattern of FMRI activation during the encoding of novel associations is differentially altered in the early stages of Alzheimer's disease compared with normal aging.
$\mathrm{T}$ he ability to consciously learn and retain new information (that is, explicit memory) is typically the earliest cognitive impairment seen in Alzheimer's disease, ${ }^{1}$ but it also decreases significantly with advancing age. ${ }^{2}$ Recent evidence suggests that the neural substrates underlying these two impairments of explicit memory differ.

There is widespread agreement that the memory impairment seen in Alzheimer's disease is the result of neuronal loss in the perforant pathway of the medial temporal lobe, a region that includes the hippocampus and the entorhinal cortex. ${ }^{3}$ Because age related neuronal loss within these brain regions is minimal, ${ }^{4}$ memory problems among non-demented older individuals are thought to be related to alterations in the coordinated modulation of other brain regions, based at least in part on decreases in neurotransmitter levels and declines in synaptic integrity. ${ }^{5}$

We therefore sought to determine whether functional magnetic resonance imaging (fMRI) data collected during an explicit encoding task would demonstrate a different pattern of activation in mild Alzheimer's disease from the pattern of alteration seen in normal aging. We chose to study associative encoding because the hippocampus is thought to play a crucial role in binding together previously unrelated items of information. ${ }^{6}$ The associative memory task we employed involved forming novel face-name associations. Such tasks appear to be particularly difficult because they require the formation of an association across the visual and verbal domains, and because first names are often arbitrarily assigned and are not conducive to forming associations. Perhaps for this reason, difficulty in remembering proper names is the most common complaint among elderly individuals and in memory disorder clinics. $^{78}$

We have previously reported that the face-name encoding task employed in this study activates a specific set of brain regions in young subjects, which include the striate, fusiform, and prefrontal cortices and the anterior hippocampus. ${ }^{9}$ In a second study with a different cohort of young subjects, we examined patterns of activation during pharmacologically induced memory impairment using lorazepam and scopolamine. In that study we demonstrated reliable activation of these regions using a two week test-retest interval, and then showed alterations in fusiform, prefrontal, and hippocampal regions during pharmacologically induced memory impairment. ${ }^{10}$ These studies suggested that there is a specific set of brain regions involved in the encoding of novel associations between names and faces. In the current study, we hypothesised that the functional alterations in this set of brain regions would differ in normal aging from that seen in mild Alzheimer's disease, and that the greatest functional differences between disease related and age related change would be evident in the hippocampus.

\section{METHODS}

\section{Subjects}

Twenty seven right handed subjects were participants in the study. They comprised three groups:

- Young: 10 young controls, mean (SD) age, 24.9 (3.5) years; age range 23 to 33 years; six female, four male;

- Elderly: 10 elderly controls, mean age 74.1 (7.3) years; age range 67 to 88 years; eight female, two male;

- Mild Alzheimer's disease: seven patients with mild probable Alzheimer's disease, mean age 80.6 (6.9) years; age range 71 to 89 years; six female, one male.

The patients with probable Alzheimer's disease were diagnosed according to NINCDS-ADRDA criteria. ${ }^{11}$ They were mildly impaired on the basis of their scores on the mini-mental state examination (MMSE) ${ }^{12}$ (mean 22.6 (2.2);

Abbreviations: $f M R I$, functional magnetic resonance imaging; $M R$, magnetic resonance; NINCDS-ADRDA, National Institute of Neurological and Communicative Disorders and Stroke-Alzheimer's Disease and Related Disorders Association; NvF, novel $v$ fixation; NvR, novel $v$ repeated; $R v F$, repeated $v$ fixation 
range 20 to 26). None of the subjects was taking prescription or over the counter drugs with central nervous system effects. All the Alzheimer patients had either been off cholinesterase inhibitors for at least 30 days before scanning or had never taken these agents. All subjects were scanned during the morning hours.

The study was approved by the human research committee of the Massachusetts General Hospital. All subjects provided written informed consent.

\section{Cognitive activation task \\ Task conditions}

The associative encoding task comprised faces (previously unfamiliar to the subjects) paired with fictional first names. The face stimuli were colour photographs taken with a digital camera with a resolution of $924 \times 1096 \mathrm{mp}$. The photographs were of individuals who varied in age (range 18 to 90 years) and ethnicity, with equal numbers of male and female faces. Popular first names were obtained from the public lists on the internet of the most commonly used names for each decade from 1910 to 1990. First names were assigned to each face by the investigators. The face stimuli were centred on a black background with the first name printed in white below the face, forming a face-name pair. Visual stimuli were presented using a Macintosh Computer (Apple) connected to a Sharp 2000 colour LCD projector. Images were projected through a collimating lens (Buhl Optical) onto a screen attached to the head coil during functional data acquisition.

Subjects were given explicit instructions to try to remember which name was associated with which face for later testing. These stimuli were used in a block design paradigm with three conditions:

- Novel: each novel face-name pair was presented once for five seconds. Subjects viewed seven novel face-name pairs during each novel block, seeing a total of 84 novel face-name pairs over the course of the session;

- Repeated: two repeated face-name pairs (one male and one female) were presented for five seconds each, with the male and female face-name pairs alternating throughout each repeated block. The two repeated face-name pairs were first shown to the subject in a practice run immediately before the functional runs, so that these stimuli were already somewhat familiar to the subjects before beginning the functional runs. Each repeated face-name pair was presented 49 times over the course of the session;

- Fixation: subjects were instructed to look at a white fixation cross $(+)$ presented in the centre of the visual field on a black background in order to focus their attention in the visual domain.

\section{Post-scan memory testing}

Immediately after scanning, subjects were tested for their memory of a subset of the face-name pairs. For this purpose, 12 faces were presented: six from the block of novel face-name pairs, four distracter faces (never presented), and the male and female faces presented throughout the repeated blocks. Subjects were asked to indicate if they had seen the face before with a yes/no answer, and if yes, to recall the name associated with the face.

\section{Image acquisition}

Structural and echo planar functional images were acquired on a 3.0 tesla General Electric scanner with ANMR upgrade (Advanced NMR Systems, Wilmington, Massachusetts, USA). The standard GE quadrature head coil was used. Subjects were positioned in the head coil with a cervical collar and foam padding to reduce head motion.

A sagittal localiser scan was done first for positioning the slice planes. A spoiled gradient echo structural scan, consisting of 60 slices (resolution $1.6 \times 1.6 \times 2.8 \mathrm{~mm}$ ), was then obtained for registration with the functional data for anatomical localisation. Functional data were then acquired using an asymmetric spin echo T2* weighted blood oxygen level dependent (BOLD) method with time of repetition (TR) $2500 \mathrm{~ms}$, time of echo (TE) $40 \mathrm{~ms}$, and flip angle $90^{\circ}$; voxel dimensions were $3.125 \times 3.125 \times 8.0 \mathrm{~mm}$. Twenty slices $(7 \mathrm{~mm}$ thick; $1 \mathrm{~mm}$ gap between slices) were acquired in a coronal orientation, perpendicular to the anterior commissureposterior commissure line. Scanning time for each functional run was 4 min $15 \mathrm{~s}$, consisting of 102 time points (four for Tl stabilisation and 98 for functional data collection). In all, six functional runs per subject were acquired.

\section{Data analysis}

Functional MRI data were preprocessed and analysed using statistical parametric mapping ${ }^{13}$ (SPM99; http:// www.fil.ion.ucl.ac.uk/spm; Wellcome Department of Cognitive Neurology) for Matlab (Mathworks Inc). The data were realigned and normalised to the standard SPM99 EPI template and then smoothed with a gaussian kernel of $8 \mathrm{~mm}$. The data were modelled with a fixed response (box-car), and convolved with the canonical haemodynamic response function. A high pass filter of 260 seconds was used for all contrasts.

Activation maps were generated for three contrasts:

- novel $v$ fixation $(\mathrm{NvF})$, which primarily provided information on the encoding of novel complex visual face-name stimuli compared with a low level visual fixation;

- repeated $v$ fixation (RvF), which primarily provided information on the viewing of familiar complex visual stimuli compared with fixation;

- novel $v$ repeated (NvR), which held the visual complexity of the stimuli constant, and thus provided information on the encoding of novel face-name associations compared with viewing familiar face-name associations.

Group averaged activation maps and between-group comparisons (young $v$ elderly controls, and elderly controls $v$ mild Alzheimer patients) were assessed using SPM 99 random effects models. ${ }^{14}$ As we chose to use random effects models and had small numbers of subjects in each group, we used a less conservative significance value than the standard whole brain corrected $\mathrm{p}$ value in SPM. Within-group activations were considered to be significant with a height threshold of 0.001 (uncorrected) and an extent threshold of at least 10 contiguous voxels, and between-group differences were considered to be significant at 0.01 (uncorrected) with at least 10 contiguous voxels. Coordinates for peak significant activations were converted from standard MNI coordinate system in SPM 99 to Talairach coordinates (http://www.mrc-cbu.cam.ac.uk/ imaging mni2tal) and localised using the neuroanatomical atlas of Talairach and Tournoux. ${ }^{15}$

In order to examine whether there was evidence of hippocampal dysfunction in addition to potential confounding effects on activation caused by atrophy, we sampled MR signal time courses within each subject's hippocampus. We placed a small $\left(6 \mathrm{~mm}^{3}\right)$ region of interest in the centre of the hippocampus, guided by each individual subject's hippocampal anatomy, and extracted the MR signal time course to assess whether there was evidence of paradigm linked activation (http://spm-toolbox.sourceforge.net).

\section{RESULTS}

\section{Within-group analyses}

The within-group activations for young controls, elderly controls, and mild Alzheimer patients for all contrasts (NvF, RvF, NvR) are shown in table 1 .

All subjects showed significant activation in striate and fusiform regions for the NvF contrast. They also all showed 
Table 1 Within-group activations (SPM99 random effects)

\begin{tabular}{|c|c|c|c|c|c|c|c|c|}
\hline \multicolumn{2}{|c|}{ Contraßregion } & BA & $\mathrm{x}$ & y & z & $\begin{array}{l}\text { No of } \\
\text { voxels }\end{array}$ & Value & $\begin{array}{l}\mathrm{p} \text { Value } \\
\text { (uncorrected) }\end{array}$ \\
\hline \multicolumn{9}{|c|}{ Young controls } \\
\hline \multirow[t]{11}{*}{ NvF } & Middle/inferior frontal gyrus & $\mathrm{BA} 9 / 46$ & 54 & 27 & 24 & 76 & 4.04 & 0.00003 \\
\hline & Middle frontal gyrus & BA 6 & -42 & 6 & 52 & 189 & 4.64 & 0.000002 \\
\hline & Inferior frontal gyrus & BA 47 & -27 & 20 & -9 & 11 & 3.42 & 0.0003 \\
\hline & Medial frontal gyrus & BA 6 & -6 & 20 & 43 & 58 & 4.62 & 0.000002 \\
\hline & Anterior cingulate & BA 33 & -6 & 7 & 19 & 18 & 3.93 & 0.00004 \\
\hline & Caudate & & -18 & -2 & 17 & 30 & 3.87 & 0.00005 \\
\hline & Thalamus & & 18 & -17 & 15 & 20 & 4.21 & 0.00001 \\
\hline & Hippocampal formation & & -24 & -24 & -11 & 104 & 4.66 & 0.000002 \\
\hline & Hippocampal formation & & 21 & -30 & -9 & 110 & 4.72 & 0.000001 \\
\hline & Extrastriate/fusiform gyrus & BA 20 & -39 & -48 & -25 & 330 & 4.4 & 0.000005 \\
\hline & Extrastriate/fusiform gyrus & BA 20 & 42 & -48 & -28 & 105 & 4.12 & 0.00002 \\
\hline \multirow[t]{4}{*}{ RvF } & Fusiform gyrus & BA 37 & 42 & -48 & -25 & 12 & 3.38 & 0.0004 \\
\hline & Extrastriate/fusiform gyrus & BA 19 & -45 & -62 & -22 & 88 & 4.5 & 0.000003 \\
\hline & Extrastriate/fusiform gyrus & BA 19 & 39 & -65 & -25 & 18 & 3.75 & 0.00009 \\
\hline & Extrastriate/lingual gyrus & BA 18 & -21 & -85 & -6 & 31 & 4.23 & 0.00001 \\
\hline \multirow[t]{11}{*}{ NvR } & Orbital frontal & BA $11 / 47$ & 27 & 31 & -14 & 39 & 4.18 & 0.00001 \\
\hline & Medial frontal gyrus & BA $6 / 8$ & -6 & 20 & 43 & 92 & 4.26 & 0.00001 \\
\hline & Inferior frontal gyrus & BA 9 & 42 & 16 & 24 & 55 & 4.11 & 0.00002 \\
\hline & Inferior frontal gyrus & BA 9 & -42 & 10 & 22 & 117 & 3.91 & 0.00005 \\
\hline & Anterior cingulate & BA 33 & 6 & 10 & 19 & 13 & 3.66 & 0.0001 \\
\hline & Middle frontal gyrus & BA 6 & -40 & 6 & 55 & 16 & 4.71 & 0.000001 \\
\hline & Hippocampal formation & BA 35 & -21 & -27 & -11 & 140 & 5.01 & 0.0000003 \\
\hline & Hippocampal formation & BA 35 & 24 & -30 & -11 & 99 & 4.94 & 0.0000004 \\
\hline & Extrastriate/fusiform gyrus & BA 20 & 42 & -48 & -28 & 103 & 4.19 & 0.00001 \\
\hline & Extrastriate/fusiform gyrus & BA 20 & -39 & -48 & -28 & 258 & 4.17 & 0.00002 \\
\hline & Angular gyrus & BA 39 & 39 & -69 & 31 & 10 & 4.02 & 0.00003 \\
\hline \multicolumn{9}{|c|}{ Elderly controls } \\
\hline \multirow[t]{11}{*}{ NvF } & Inferior frontal gyrus & BA 45 & 51 & 38 & 9 & 53 & 3.86 & 0.00006 \\
\hline & Inferior frontal gyrus & BA 47 & -36 & 29 & -6 & 12 & 3.42 & 0.0003 \\
\hline & Inferior frontal gyrus & BA 45 & -54 & 21 & 13 & 17 & 3.62 & 0.0001 \\
\hline & Inferior frontal gyrus & BA 9 & 53 & 10 & 33 & 56 & 3.75 & 0.00009 \\
\hline & Anterior cingulate & BA 33 & -3 & 10 & 19 & 13 & 4.25 & 0.00001 \\
\hline & Putamen & & -21 & -8 & 9 & 18 & 4.06 & 0.00002 \\
\hline & Hippocampal formation & & -21 & -27 & -14 & 84 & 4.3 & 0.000008 \\
\hline & Hippocampal formation & & 21 & -24 & -14 & 30 & 3.98 & 0.00004 \\
\hline & Extrastriate/fusiform gyrus & BA 37 & 54 & -56 & -17 & 65 & 4.55 & 0.000003 \\
\hline & Extrastriate/fusiform gyrus & BA 37 & -45 & -57 & -22 & 345 & 5.23 & 0.00000009 \\
\hline & Superior parietal lobule & BA 7 & -27 & -59 & 44 & 55 & 3.57 & 0.0002 \\
\hline \multirow[t]{9}{*}{ RvF } & Inferior frontal gyrus & BA $44 / 45$ & -42 & 30 & 12 & 36 & 3.8 & 0.00007 \\
\hline & Hippocampal formation & & 27 & -18 & -14 & 38 & 4.18 & 0.00001 \\
\hline & Inferior parietal lobule & BA 40 & -27 & -59 & 36 & 11 & 3.43 & 0.0003 \\
\hline & Inferior parietal lobule & BA 40 & 42 & -50 & 47 & 15 & 3.68 & 0.0001 \\
\hline & Superior parietal lobule & BA 7 & -33 & -50 & 52 & 18 & 3.56 & 0.0002 \\
\hline & Superior parietal lobule & BA 7 & -30 & -74 & 42 & 12 & 3.67 & 0.0001 \\
\hline & Extrastriate/fusiform gyrus & BA 37 & -45 & -54 & -23 & 94 & 4.47 & 0.000004 \\
\hline & Extrastriate/fusiform gyrus & BA 37 & 48 & -54 & -20 & 29 & 4.12 & 0.00002 \\
\hline & Middle temporal gyrus & BA 19 & -33 & -78 & 18 & 23 & 3.51 & 0.0002 \\
\hline \multirow[t]{5}{*}{ NvR } & Anterior cingulate & BA 33 & -3 & 10 & 22 & 17 & 4 & 0.00003 \\
\hline & Putamen & & -21 & -11 & 9 & 16 & 3.53 & 0.0002 \\
\hline & Hippocampal formation & BA 28 & -27 & -24 & -6 & 58 & 4.36 & 0.000006 \\
\hline & Hippocampal formation & BA 28 & 15 & -24 & -14 & 17 & 3.58 & 0.0002 \\
\hline & Extrastriate/fusiform gyrus & BA 37 & -39 & -45 & -26 & 251 & 4.61 & 0.000002 \\
\hline \multicolumn{9}{|c|}{ Mild Alzheimer's disease } \\
\hline \multirow[t]{7}{*}{ NvF } & Inferior frontal gyrus & BA 9 & -48 & 16 & 24 & 75 & 3.71 & 0.0001 \\
\hline & Inferior frontal gyrus & BA 9 & 48 & 13 & 32 & 21 & 3.66 & 0.0001 \\
\hline & Hippocampal formation & BA 28 & 21 & -27 & -9 & 19 & 3.65 & 0.0001 \\
\hline & Precuneus/superior parietal lobule & BA 7 & 18 & -56 & 47 & 74 & 4.38 & 0.000006 \\
\hline & Superior parietal lobule & $\mathrm{BA} 7$ & -33 & -59 & 47 & 21 & 3.58 & 0.0002 \\
\hline & Extrastriate/fusiform gyrus & BA 37 & -27 & -45 & -18 & 50 & 3.82 & 0.00007 \\
\hline & Extrastriate/fusiform gyrus & BA 19 & 15 & -67 & -9 & 23 & 3.92 & 0.0004 \\
\hline RvF & No significant clusters found & & & & & & & \\
\hline NvR & Fusiform gyrus & BA 37 & 9 & -44 & -8 & 10 & 3.5 & 0.0002 \\
\hline
\end{tabular}


significant activation in prefrontal and superior parietal regions, but young and elderly controls differed in the pattern of activation in these regions, which is discussed in detail below.

Both young and elderly controls showed significant paradigm linked activation in the hippocampal formation in both the NvF and NvR contrast. In the NvR contrast, the hippocampal activation was greater in extent and more symmetrical in the young controls than in the elderly controls; however, the location and significance of the peak activation within the hippocampus was similar (within-group random effects averages: peak voxel location, right: $24,-30,-11$ $(\mathrm{p}<0.0001)$; left: $-2 \mathrm{l},-27,-1 \mathrm{l}(\mathrm{p}<0.0001)$ for young controls; right: $15,-24,-14(\mathrm{p}=0.0002)$; left: $-27,-24,-6$ $(p<0.0001)$ for elderly controls $)$. The patients with mild Alzheimer's disease showed no activation in either hippocampal formation for the NvR contrast, and only a small area of activation in the right hippocampal formation for the NvF contrast $(21,-27,-9(p=0.0001))$. In addition, sampling of the MR signal within the hippocampus using a region of interest analysis showed that only one of the seven Alzheimer patients had evidence of paradigm linked MR signal within the hippocampus (fig 1). This patient had the mildest dementia in the group (age $=84 ;$ MMSE $=26$ ).

\section{Between-group analyses}

The between-group differences for both young $v$ elderly controls and elderly controls $v$ mild Alzheimer's disease for all contrasts (NvF, RvF, NvR) are shown in table 2.

\section{Young $v$ elderly controls}

The most significant differences between the young and elderly controls were found in the pattern of activation in prefrontal and parietal cortices (fig 2). In the NvF contrast, young controls showed greater activation than elderly controls in left superior prefrontal regions (BA 10, peak coordinates $-18,53$, 17; random effects between-group difference, $\mathrm{p}=0.0002$ ). Conversely, elderly controls showed greater activation than the young controls in superior and inferior parietal regions bilaterally (BA 7, peak coordinates: $18,-65,50(\mathrm{p}=0.0003)$, and $-18,-71,31(\mathrm{p}=0.0004) ;$ BA 40, peak coordinates: $42,-36,29$ $(\mathrm{p}=0.0003)$, and $-50,-22,29(\mathrm{p}=0.0005))$, and also in the anterior cingulate region $(-9,30,12(\mathrm{p}=0.0002))$ for the NvF contrast. The elderly controls also showed greater activation than the young controls in multiple regions for the RvF contrast, particularly in the prefrontal regions (BA 8/9: -30 , $14,49(\mathrm{p}=0.0002))$ and the superior parietal regions (BA 7: $-33,-50,49(\mathrm{p}=0.0004))$.

There were no significant differences between the young controls and the elderly controls in hippocampal activation for the NvF contrast. In the NvR contrast, there were no differences in the left hippocampal formation, but young subjects showed greater activation in the right hippocampal formation. In the RvF contrast, elderly subjects showed slightly greater activation in the right hippocampal formation $(27,-18,-14(\mathrm{p}=0.003))$. Young and elderly controls showed a similar pattern of significant paradigm linked activation in striate and fusiform cortices in all contrasts, but the young subjects showed a greater extent of activation than the elderly controls in the posterior extrastriate regions (BA 18/19: $-9,-64,6(p=0.003))$.

\section{Elderly controls $v$ mild Alzheimer patients}

The most significant differences between elderly controls and mild Alzheimer patients were seen in the hippocampal formation for both the NvF and NvR contrasts. The patients with Alzheimer's disease showed significantly less activation than elderly controls for the NvF contrasts bilaterally in the hippocampal formation (random effects between-group difference: $12,-33,-13(\mathrm{p}=0.001)$ and $-30,-24,-4$ $(p=0.003))$. For the NvR contrast, the only region showing a significant decrease in the Alzheimer group compared with
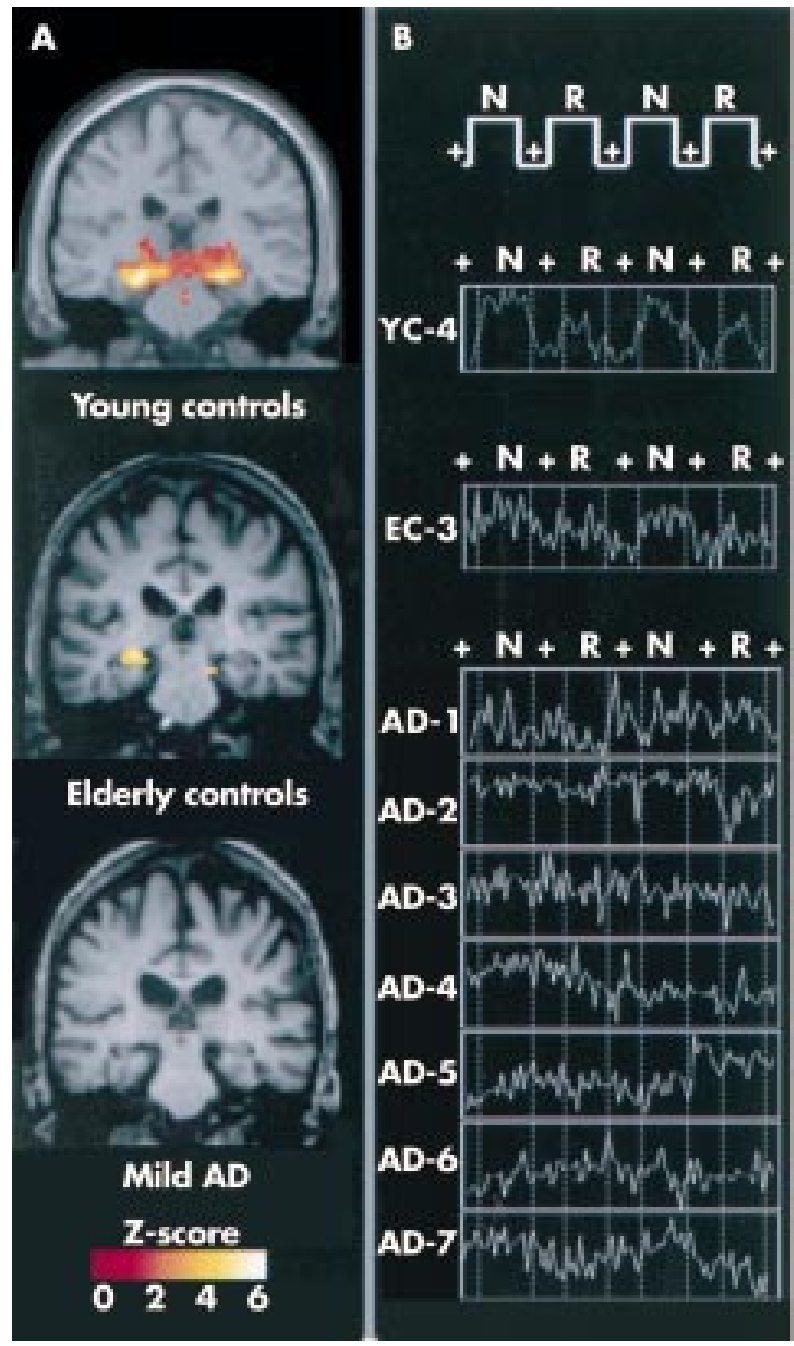

Figure 1 (A) Within-group, random effects average activation maps for the novel $v$ repeated contrast for young controls, elderly controls, and patients with mild Alzheimer's disease, shown on a representative structural image from each group in the coronal plane at the level of the hippocampal formation. (B) Magnetic resonance signal time courses on a standardised scale from a representative young control (YC-4), a representative elderly control (EC-3), and the seven patients with mild Alzheimer's disease. Only one Alzheimer patient (AD-7; MMSE = 26) showed evidence of paradigm linked activation within the hippocampus.

the elderly controls was located in the right hippocampal formation $(15,-24,-16(\mathrm{p}=0.002))$.

The elderly controls and the Alzheimer patients showed a similar pattern of significant activation in striate and posterior fusiform cortices. Interestingly, there were several neocortical regions that showed increased activation in the Alzheimer group compared with the elderly controls. These increases were most evident in the medial parietal cortex bilaterally in NvF and NvR (precuneus: $-15,-66,28(\mathrm{p}=0.0004))$, the right posterior cingulate in NvF and NvR $(12,-37,21(p<0.001))$, and the superior frontal cortex in NvF, RvF, and NvR (BA 9: $-24,48,28(\mathrm{p}<0.0001))$.

\section{Post-scan testing}

Young controls correctly identified $94 \%$ of the faces in post-scan testing, compared with $78 \%$ correct for the elderly controls $(p<0.05)$. The young controls correctly named $58 \%$ of the faces on free recall of the name, compared with $40 \%$ for the elderly controls $(p<0.05)$. All young and elderly controls correctly identified both repeated faces. All young subjects 
Table 2 Between-group differences (SPM99 random effects)

\begin{tabular}{|c|c|c|c|c|c|c|c|c|}
\hline \multicolumn{2}{|c|}{ ContrastRegion } & BA & $x$ & $y$ & $z$ & $\begin{array}{l}\text { No of } \\
\text { voxels }\end{array}$ & zalue & $\begin{array}{l}\text { p Value } \\
\text { (uncorrected) }\end{array}$ \\
\hline \multicolumn{9}{|c|}{ Young control > elderly control } \\
\hline \multirow[t]{5}{*}{$\mathrm{NvF}$} & Superior frontal gyrus & BA 10 & -18 & 53 & 17 & 80 & 3.49 & 0.0002 \\
\hline & Superior frontal gyrus & BA 10 & 18 & 62 & 24 & 12 & 2.43 & 0.008 \\
\hline & Inferior frontal gyrus & BA 46 & -45 & 47 & 3 & 23 & 3.2 & 0.001 \\
\hline & Extrastriate/lingual gyrus & BA 18 & -9 & -64 & 6 & 25 & 2.76 & 0.003 \\
\hline & Extrastriate/lingual gyrus & BA 19 & 33 & -67 & -4 & 91 & 3.29 & 0.001 \\
\hline RvF & No significant clusters & & & & & & & \\
\hline \multirow[t]{8}{*}{ NvR } & Inferior frontal gyrus & BA 46 & -45 & 47 & 3 & 79 & 3.35 & 0.0004 \\
\hline & Middle frontal gyrus & BA 9 & -42 & 13 & 24 & 12 & 2.72 & 0.003 \\
\hline & Caudate & & 21 & 29 & 4 & 13 & 2.74 & 0.003 \\
\hline & Caudate & & 9 & 12 & -1 & 17 & 3.04 & 0.001 \\
\hline & Thalamus & & 0 & -18 & -4 & 159 & 3.1 & 0.001 \\
\hline & Hippocampal formation & & 30 & -24 & -11 & 62 & 3.39 & 0.0003 \\
\hline & Extrastriate/lingual gyrus & BA 18 & -15 & -64 & 3 & 464 & 3.63 & 0.0001 \\
\hline & Extrastriate/lingual gyrus & BA 18 & 6 & -70 & -7 & 188 & 3.09 & 0.001 \\
\hline \multicolumn{9}{|c|}{ Elderly control > young control } \\
\hline \multirow[t]{12}{*}{ NvF } & Anterior cingulate & BA 24 & -9 & 30 & 12 & 50 & 3.55 & 0.0002 \\
\hline & Cingulate gyrus & BA 24 & 12 & 13 & 32 & 46 & 2.78 & 0.003 \\
\hline & Cingulate gyrus & BA 24 & 3 & -4 & 39 & 41 & 3.22 & 0.001 \\
\hline & Inferior frontal gyrus & BA 9 & 24 & 10 & 33 & 24 & 3.1 & 0.001 \\
\hline & Inferior frontal gyrus & BA 9 & 60 & 7 & 22 & 123 & 3.13 & 0.001 \\
\hline & Middle frontal gyrus & BA 6 & 24 & 5 & 47 & 42 & 3.2 & 0.001 \\
\hline & Superior temporal gyrus & BA 38 & 56 & 14 & -16 & 14 & 3.18 & 0.001 \\
\hline & Superior temporal gyrus & BA 38 & -45 & -21 & -7 & 51 & 2.93 & 0.002 \\
\hline & Inferior parietal lobule & BA 40 & -50 & -22 & 29 & 160 & 3.3 & 0.0005 \\
\hline & Inferior parietal lobule & BA 40 & 42 & -36 & 29 & 175 & 3.45 & 0.0003 \\
\hline & Superior parietal lobule & $\mathrm{BA} 7$ & 18 & -65 & 50 & 63 & 3.47 & 0.0003 \\
\hline & Superior parietal lobule & $\mathrm{BA} 7$ & -18 & -71 & 31 & 181 & 3.37 & 0.0004 \\
\hline \multirow[t]{12}{*}{ RvF } & Middle frontal gyrus & BA 11 & -30 & 31 & -19 & 102 & 3.51 & 0.0002 \\
\hline & Inferior frontal gyrus & BA 47 & 36 & 29 & -6 & 91 & 3.5 & 0.0002 \\
\hline & Inferior frontal gyrus & BA 9 & 24 & 10 & 33 & 150 & 3.87 & 0.0006 \\
\hline & Inferior frontal gyrus & BA 44 & 53 & 6 & 8 & 20 & 2.77 & 0.003 \\
\hline & Superior frontal gyrus & BA 8 & -30 & 14 & 49 & 782 & 4.16 & 0.0002 \\
\hline & Thalamus & & 0 & 9 & 2 & 187 & 3.76 & 0.0008 \\
\hline & Hippocampal formation & & 27 & -18 & -14 & 24 & 2.8 & 0.003 \\
\hline & Cingulate gyrus & BA 31 & -12 & -27 & 43 & 20 & 2.86 & 0.002 \\
\hline & Fusiform gyrus & BA 20 & 45 & -30 & -14 & 44 & 3.12 & 0.001 \\
\hline & Fusiform gyrus & BA 20 & 33 & -39 & -13 & 16 & 2.71 & 0.003 \\
\hline & Middle temporal gyrus & BA 21 & -50 & -47 & 2 & 56 & 4.1 & 0.0002 \\
\hline & Superior parietal lobule & $\mathrm{BA} 7$ & -33 & -50 & 49 & 1459 & 3.95 & 0.0004 \\
\hline \multirow[t]{2}{*}{ NvR } & Superior temporal gyrus & BA 6 & -48 & -3 & 6 & 15 & 2.58 & 0.005 \\
\hline & Postcentral gyrus & BA 3 & 60 & -11 & 23 & 14 & 2.54 & 0.006 \\
\hline \multicolumn{9}{|c|}{ Elderly control > Alzheimer's disease } \\
\hline \multirow[t]{4}{*}{$\mathrm{NvF}$} & Inferior frontal gyrus & BA 45 & 54 & 36 & 9 & 12 & 2.93 & 0.002 \\
\hline & Hippocampal formation & & -30 & -24 & -4 & 11 & 2.78 & 0.003 \\
\hline & Hippocampal formation & & 12 & -33 & -13 & 63 & 2.99 & 0.001 \\
\hline & Superior parietal lobule & BA 7 & -21 & -60 & 42 & 12 & 2.57 & 0.005 \\
\hline RvF & Middle frontal gyrus & BA 11 & 27 & 42 & -13 & 58 & 2.75 & 0.003 \\
\hline & Middle frontal gyrus & BA 6 & -30 & 14 & 46 & 34 & 2.88 & 0.002 \\
\hline & Inferior frontal gyrus & BA 47 & -18 & 37 & -9 & 13 & 2.9 & 0.002 \\
\hline & Anterior cingulate & BA 24 & -12 & 32 & -1 & 15 & 3.03 & 0.001 \\
\hline & Putamen & & 24 & 6 & 5 & 60 & 2.71 & 0.003 \\
\hline & Hippocampal formation & & 27 & -18 & -14 & 10 & 3.09 & 0.001 \\
\hline & Hippocampal formation & & -33 & -27 & -19 & 18 & 3.08 & 0.001 \\
\hline & Middle temporal gyrus & BA 39 & 36 & -54 & 30 & 27 & 2.99 & 0.001 \\
\hline & Middle temporal gyrus & BA 39 & -36 & -75 & 23 & 166 & 3.87 & 0.0001 \\
\hline & Middle occipital gyrus & BA 19 & 30 & -70 & 9 & 46 & 2.97 & 0.001 \\
\hline NvR & Hippocampal formation & & 15 & -24 & -16 & 24 & 2.86 & 0.002 \\
\hline Alzh & mer's disease > elderly c & ntrol & & & & & & \\
\hline $\mathrm{NvF}$ & Superior frontal gyrus & BA 9 & -24 & 48 & 28 & 50 & 3.27 & 0.0001 \\
\hline & Middle temporal gyrus & BA 21 & -42 & -15 & -9 & 10 & 2.72 & 0.003 \\
\hline & Superior temporal gyrus & BA 41 & 56 & -25 & 10 & 20 & 2.77 & 0.003 \\
\hline & Posterior cingulate & BA 29 & 12 & -37 & 21 & 18 & 3.17 & 0.001 \\
\hline & Precuneus & BA 31 & 9 & -62 & 34 & 108 & 3.65 & 0.0001 \\
\hline & Precuneus & BA 31 & -15 & -69 & 28 & 57 & 3.46 & 0.0003 \\
\hline RvF & Superior frontal gyrus & BA 6 & -9 & 26 & 57 & 10 & 3.9 & 0.00005 \\
\hline NvR & Superior frontal gyrus & BA 9 & -27 & 48 & 28 & 35 & 3.48 & 0.0002 \\
\hline & Superior frontal gyrus & BA 9 & -15 & 40 & 37 & 11 & 2.54 & 0.006 \\
\hline
\end{tabular}


Table 2 continued

\begin{tabular}{|c|c|c|c|c|c|c|c|c|}
\hline \multicolumn{2}{|c|}{ ContrastRegion } & \multirow{2}{*}{$\begin{array}{l}\text { BA } \\
\text { BA } 10\end{array}$} & \multirow{2}{*}{$\frac{x}{-21}$} & \multirow{2}{*}{$\frac{y}{35}$} & \multirow{2}{*}{$\frac{z}{1}$} & \multirow{2}{*}{$\begin{array}{c}\text { No of } \\
\text { voxels }\end{array}$} & \multirow{2}{*}{$\begin{array}{l}\text { Value } \\
3.3\end{array}$} & \multirow{2}{*}{$\begin{array}{l}\begin{array}{l}\text { p Value } \\
\text { (uncorrected) }\end{array} \\
0.0005\end{array}$} \\
\hline NvR & Cingulate & & & & & & & \\
\hline & Middle temporal gyrus & BA 21 & 50 & -4 & -12 & 20 & 2.78 & 0.003 \\
\hline & Middle temporal gyrus & BA 21 & -48 & -9 & -15 & 11 & 2.81 & 0.002 \\
\hline & Precuneus/posterior cingulate & BA 31 & 12 & -57 & 33 & 443 & 3.6 & 0.0002 \\
\hline & Fusiform gyrus & BA 36 & -36 & -27 & -19 & 22 & 2.99 & 0.001 \\
\hline & Precuneus & BA 31 & -15 & -66 & 28 & 83 & 3.35 & 0.0004 \\
\hline & Extrastriate/fusiform gyrus & BA 18 & -21 & -76 & -4 & 31 & 2.89 & 0.002 \\
\hline
\end{tabular}

correctly recalled the names of both repeated faces, while two elderly subjects missed one of the names of the repeated faces on free recall, which was not a significant difference.

The patients with mild Alzheimer's disease correctly recognised $60 \%$ of the faces, a difference from elderly controls that approached significance $(p=0.059)$. The Alzheimer patients named $12 \%$ of the faces correctly, which was significantly less than elderly controls $(p<0.005)$. Five of the Alzheimer group correctly recognised both of the repeated faces, while two correctly recognised only one of the repeated faces, which was a trend towards a significant difference from the elderly controls $(p=0.08)$. The Alzheimer patients performed significantly worse than the elderly controls on free recall of the names for the repeated faces $(p<0.006)$.

\section{DISCUSSION}

These findings suggest that the functional neuroanatomical alterations underlying explicit memory changes in mild Alzheimer's disease differ from those seen with normal aging. Particularly striking was the fact that the regions showing the greatest decreases in activation in the patients with mild Alzheimer's disease compared with the elderly controls were in the hippocampal formation. We hypothesise that this is the result of the extensive neuronal loss (in conjunction with neuritic plaques and neurofibrillary tangles) that develops early in the course of Alzheimer's disease. ${ }^{3}$ It is likely that regional atrophy is also at least partially responsible for the decreased hippocampal activation in Alzheimer's disease.$^{16}$ However, this is unlikely to be the entire explanation for our findings, as we saw little evidence of paradigm linked activation in the hippocampus in six of the seven Alzheimer patients when the MR signal was sampled within a small section of the hippocampus, guided by each individual subject's anatomy. This suggests that

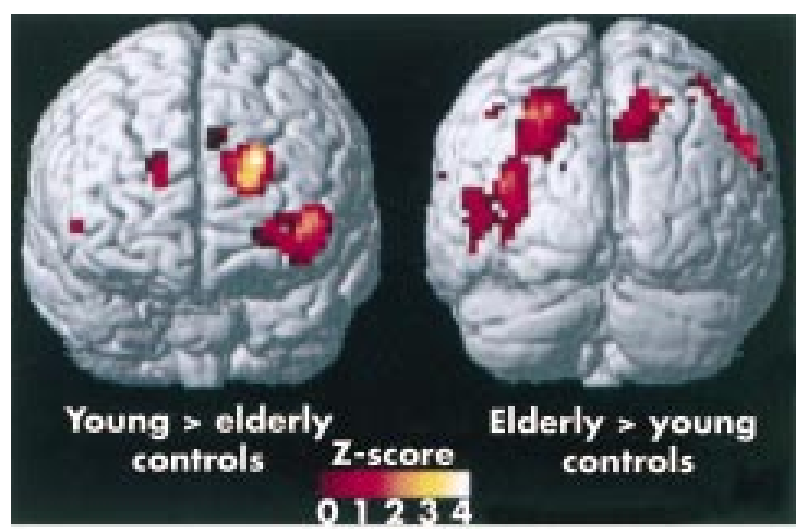

Figure 2 Between-group random effects comparison for young $v$ elderly controls for the novel $v$ fixation contrast. Young controls showed greater activation than elderly controls (shown on the left) in the prefrontal regions, whereas elderly controls showed greater activation than young controls in the superior parietal regions (shown on the right). in addition to hippocampal atrophy, there is dysfunction in the remaining hippocampal tissue. We studied fewer Alzheimer patients than elderly controls; however, several regions showed greater activation in the Alzheimer group than in the elderly controls, suggesting that reduced power is also unlikely to explain the findings in the hippocampus. Further fMRI studies with larger numbers of subjects are clearly needed; however, our findings are consistent with the few fMRI studies using encoding tasks in Alzheimer's disease published to date, ${ }^{17-19}$ and suggest that fMRI may be useful in assessing the integrity of hippocampal function early in the course of the disease.

Our findings with respect to elderly $v$ young controls showed a different anatomical distribution than in the elderly $v$ Alzheimer's disease comparison discussed above. The elderly controls showed significant hippocampal activation which was very similar to young controls for the NvF contrast. For the NvR contrast, the activation of the hippocampus was similar in magnitude between the young and elderly controls, but was greater in extent and more symmetrical in the young controls. These findings are consistent with reports of minimal cell loss accompanied by alterations in the neurotransmitter levels and synaptic integrity within the hippocampus in normal aging. ${ }^{45}$ Consistent with our results, recent fMRI studies using encoding tasks have reported significant activation within the hippocampal formation in elderly controls, ${ }^{17-20}$ but have also reported age related decreases in hippocampal activation during an associative working memory task. ${ }^{21}$

The most striking age related change in the present study was the alteration in the pattern of prefrontal and parietal activation seen in the elderly controls compared with the young controls. The elderly controls showed decreased prefrontal activation and increased parietal activation during the encoding of novel facename pairs compared with the young controls. We hypothesise that these alterations result in differential activity in a frontoparietal attentional network, which may in part underlie age related changes in memory. These findings are consistent with several recent fMRI and positron emission tomography (PET) studies showing age related alterations in patterns of prefrontal activation during memory tasks. ${ }^{22-25}$

It is noteworthy that the patients with mild Alzheimer's disease showed greater activation in several neocortical regions than the elderly controls, as mentioned above. The most striking increases were found in medial parietal cortex (precuneus) and posterior cingulate. These regions have been reported to show decreased resting metabolism and perfusion in patients with mild Alzheimer's disease and in asymptomatic genetically at risk subjects. ${ }^{26-28}$ Our findings suggest that these regions-which show evidence of dysfunction at restmay have an exaggerated increase in activation during an encoding task. This could be the result of a compensatory process that occurs once there is substantial neuronal loss within the hippocampus. In an effort to perform the task, patients with Alzheimer's disease may engage other neural systems, resulting in increased activation in these brain regions compared with elderly controls. Previous PET activation studies in Alzheimer's disease, ${ }^{29}{ }^{30}$ and a recent fMRI 
study in genetically at risk subjects, ${ }^{20}$ have likewise suggested such a compensatory mechanism.

It is also possible, however, that the strategies employed to learn the face-name associations differed among the groups of subjects. Although all subjects were instructed at the start of each run to try to remember which name was associated with each face during the presentation of the face-name pairs and to look at the fixation cross during the fixation condition, it is possible that some subjects were rehearsing the face-name pairs during fixation, which could account for some of the group differences in the patterns of activation in the NvF and RvF contrast. Our fMRI findings, however, are consistent with the differences seen in behavioural performance on the post-scan memory tests. Elderly subjects performed significantly worse than young subjects on novel face recognition and free recall of the names. The patients with Alzheimer's disease performed significantly worse than the elderly controls on novel face recognition and on novel and repeated free recall of the name.

Finally, it should be noted that although the young subjects showed a greater extent of activation in the posterior extrastriate regions, young and elderly controls and Alzheimer patients showed similar patterns of significant paradigm linked activation in the striate and extrastriate cortices. These results indicate that both elderly controls and people with Alzheimer's disease are able to mount a significant BOLD response to stimuli, and furthermore, that these extrastriate regions are responding appropriately to the presentation of complex visual stimuli, consistent with the absence of pathology in these regions in aging or in mild Alzheimer's disease. ${ }^{3}$

\section{Conclusions}

In summary, our study indicates that the pattern of activation involved during the encoding of novel associations is differentially altered in the early stages of Alzheimer's disease compared with the alterations seen in normal aging. Age related changes in memory performance appear to be related to alterations in the frontoparietal regions involved in complex attention, whereas the changes related to Alzheimer's disease seem to reflect alterations in the hippocampus and related regions involved in medial temporal memory systems. Our results must be interpreted with some caution, given the small number of subjects in each group, and hence the use of less stringent corrections for multiple comparisons. Further fMRI studies with larger numbers of elderly control subjects, subjects with mild cognitive impairment, and patients with Alzheimer's disease are under way to clarify the earliest point in the course of Alzheimer's disease when such differences can be observed.

\section{ACKNOWLEDGEMENTS}

We gratefully acknowledge the contribution of the study subjects, the Alzheimer's disease patients, and their caregivers. We thank Terry Campbell and Mary Foley for assistance with scan acquisition. This work was supported by grants from NINDS: K23-NS02189 (RAS), NIMH: MH6094l (DLS), NIA: AG08441 (DLS); P01-AG04953 (MSA), and the Dana Foundation (MSA)

\section{Authors' affiliations}

R A Sperling, E F Chua, A J Cocchiarella, D M Rentz, Memory Disorders Unit, Department of Neurology, Brigham and Women's Hospital, Boston, Massachusetts, USA

J F Bates, B R Rosen, NMR Center, Massachusetts General Hospital, Boston

M S Albert, Gerontology Research Unit, Department of Psychiatry, Massachusetts General Hospital, Boston

D L Schacter, Department of Psychology, Harvard University, Cambridge, Massachusetts

Competing interests: none declared

\section{REFERENCES}

1 Welsh K, Butters N, Hughes J, et al. Detection of abnormal memory decline in mild cases of Alzheimer's disease using CERAD neuropsychological measures. Arch Neurol 1991;48:278-81.

2 Craik F. Age differences in human memory. In: Birren J, Schaie K, eds. Handbook of the psychology of aging. New York: Van Nostrand Reinhold, 1977:384-420.

3 Braak H, Braak E. Neuropathological staging of Alzheimer-related changes. Acta Neuropathol 1991;82:239-59.

4 Morrison JH, Hof PR. Life and death of neurons in the aging brain. Science 1997;278:412-19.

5 Smith TD, Adams MM, Gallagher M, et al. Circuit-specific alterations in hippocampal synaptophysin immunoreactivity predict spatial learning impairment in aged rats. J Neurosci 2000;20:6587-93.

6 Eichenbaum H. How does the brain organize memories? Science 1997;277:333-5.

7 Zelinski EM, Gilewski M. Assessment of memory complaints by rating scales and questionnaires. Psychopharmacol Bull 1988;24:523-9.

8 Leirer VO, Morrow DG, Sheikh Jl, et al. Memory skills elders want to improve. Exp Aging Res 1990;16:155-8.

9 Sperling R, Bates J, Cocchiarella A, et al. Encoding novel face-name associations: a functional MRI study. Hum Brain Map 2001;14:129-39.

10 Sperling R, Greve D, Dale A, et al. fMRI detection of pharmacologically induced memory impairment. Proc Natl Acad Sci USA 2002;99:455-60

11 McKhann G, Drachman D, Folstein M, et al. Clinical diagnosis of Alzheimer's disease: report of the NINCDS-ADRDA work group under the auspices of Department of Health and Human Services task force on Alzheimer's disease. Neurology 1984;34:939-44.

12 Folstein MF, Folstein SE, McHugh PR. "Mini-mental state". A practical method for grading the cognitive state of patients for the clinician. $J$ Psychiatr Res 1975;12:189-98.

13 Friston KJ, Holmes AP, Worsley KJ, et al. Statistical parametric maps in functional imaging: a general linear model approach. Human Brain Mapping 1995;2:189-210.

14 Holmes AP, Friston KJ. Generalisability, random effects, and population inference. Neuroimage 1998;7:S754

15 Talairach J, Tournoux P. Co-planar stereotaxic atlas of the human brain Stuttgart: Thieme, 1988

16 Jack CR, Petersen RC, Xu YC, et al. Medial temporal atrophy on MRI in normal aging and very mild Alzheimer's disease. Neurology 1997;49:786-94

17 Small SA, Perera GM, DeLaPaz R, et al. Differential regional dysfunction of the hippocampal formation among elderly with memory decline and Alzheimer's disease. Ann Neurol 1999;45:466-72.

18 Rombouts SA, Barkhof F, Veltman DJ, et al. Functional MR imaging in Alzheimer's disease during memory encoding. Am J Neuroradiol 2000;21:1869-75.

19 Kato T, Knopman D, Liu H. Dissociation of regional activation in mild AD during visual encoding: a functional MRI study. Neurology 2001;57:812-16.

20 Bookheimer SY, Strojwas MH, Cohen MS, et al. Patterns of brain activation in people at risk for Alzheimer's disease. N Engl J Med 2000;343:450-6.

21 Mitchell KJ, Johnson MK, Raye CL, et al. FMRI evidence of age-related hippocampal dysfunction in feature binding in working memory. Brain Res Cogn Brain Res 2000; 10:197-206.

22 Cabeza R, Anderson N, Houle S, et al. Age-related differences in neural activity during item and temporal-order memory retrieval: a positron emission tomography study. J Cogn Neurosci 2000;1 2:197-206.

23 Rypma B, D'Esposito M. Isolating the neural mechanisms of age-related changes in human working memory. Nat Neurosci 2000;3:509-15.

24 Rypma B, Prabhakaran V, Desmond JE, et al. Age differences in prefrontal cortical activity in working memory. Psychol Aging $2001 ; 16: 371-84$.

25 Langley LK, Madden DJ. Functional neuroimaging of memory: implications for cognitive aging. Microsc Res Techn 2000;51:75-84.

26 Small GW, Ercoli LM, Silverman DH, et al. Cerebral metabolic and cognitive decline in persons at genetic risk for Alzheimer's disease. Proc Natl Acad Sci USA 2000;97:6037-42.

27 Reiman EM, Caselli R, Chen K, et al. Declining brain activity in cognitively normal apolipoprotein $\mathrm{E}$ varepsilon 4 heterozygotes: a foundation for using positron emission tomography to efficiently test treatments to prevent Alzheimer's disease. Proc Natl Acad Sci USA $2001 ; 98: 3334-9$.

28 Johnson KA, Lopera F, Jones K, et al. Presenilin-1-associated abnormalities in regional cerebral perfusion. Neurology 2001;56:1545-51.

29 Becker JT, Mintum MA, Aleva K, et al. Compensatory reallocation of brain resources supporting verbal episodic memory in Alzheimer's disease. Neurology 1996;46:692-700.

30 Stern Y, Moeller JR, Anderson K, et al. Different brain networks mediate task performance in normal aging and $A D$ : defining compensation. Neurology 2000;55:1291-7. 